\title{
Detection of Plant Leaf Disease Employing Image Processing and Gaussian Smoothing Approach
}

\author{
Isaac Kofi Nti \\ Department of \\ Electrical/Electronic \\ Engineering \\ Sunyani Technical University \\ Sunyani, Ghana
}

\author{
Gyamfi Eric \\ Department of \\ Electrical/Electronic \\ Engineering \\ Sunyani Technical University \\ Sunyani, Ghana
}

\author{
Yeboah Samuel Jonas \\ Department of \\ Electrical/Electronic \\ Engineering \\ Sunyani Technical University \\ Sunyani, Ghana
}

\begin{abstract}
A study of plant observation is critical to regulate the unfold of illness in plants, but its value could be higher and as a result, the producers of agricultural products often skip important preventive procedures to keep their production cost at low value. The detection of plant leaf is a vital factor to forestall serious natural event. Most plant diseases are caused by bacteria, fungi, and viruses. An automatic detection of plant disease is a necessary analytical topic. Computer vision techniques are used to uncover the affected spots from the image through an image processing technique capable of recognizing the plant lesion options is delineated in this paper. The achieved accuracy of the overall system is $90.96 \%$, in line with the experimental results.
\end{abstract}

\section{Keywords}

Digital-Pictures, Matlab, Image-Processing, Segmentation, Plant-Leaf-Diseases, agricultural-production

\section{INTRODUCTION}

Agriculture has become much a lot of than merely a way to nurse ever growing populaces. Plants have become a vital source of energy, and are a basic piece in the puzzle to resolve the matter of world warming [1]. Diseases in plants increases the value of agricultural production and a total economic disaster of producers if not cured appropriately at early stages [2] and [3]. This can impact negative on the country whose economic income is solely depends on agricultural produces. Procedures needs to monitor their plants regularly and observe any primary symptoms so as to prevent the unfold of a plant sickness, with low cost and save the major part of the production. In 2015 Ghana lose 30\% of its annual crop yields to pests and bug infestation, the situation, was attributed to restricted access to plant health services as a result of inadequate extension officers [4]. Engagement of professional agriculturists might be expensive particularly in remote isolated geographic regions. Traditionally most farmers adopts eye observation as the main method of identifying diseases among their crops and produces. This approach becomes tiresome when farm lands becomes large. But the demand of this methodology is continuous observation of the sector by an individual having superior data regarding the plants and its corresponding diseases. Moreover, appointing such a person would may prove pricey [5]. An alternative methodology is seeking advice from the professional by farmers when signs of diseases crop up, and the expert advice should come in time otherwise it could ends up in loss. Diagnosis of illness on plant will conjointly be tired laboratory testing. But this technique needs satisfactory laboratory conditions on with skilled data. The pathogen detection ways will offer a lot of correct results. As the tests are dole out of field the value could also be high and will be time intense [5]. An artificial intelligence and Machine vision can offer a best and alternative way for plant monitoring and early detection of disease, which can be control by professional from a distance and offer their professional advice at a low cost [2]. The self-recognition of the sickness is based mostly on the identification of the symptoms of disease. So that data concerning the sickness incidence may be quickly and accurately provided to the farmers, experts and researchers, this in turn reduces the watching of enormous field by soul [5].

This paper proposed a system which will detects, identify diseases on crops and offer extra classification of malady. It tries to replace the necessity of the experts to bound extent. Here, the captured image of affected leaf is first pre-processed with an image processing algorithm enhance with Gaussian smoothing operator is a 2-D sophistication operator for smoothened images and remove detail (higher frequencies) and noise. The enchased image is segmented and features extracted for a final statistical analysis for classifying the malady occurred on the leaf.

\section{IMAGE PROCESSING TECHNIQUES}

An image is the visual depiction of an object lit up by a radiation source and all Image formation has the following essentials, an object, a particle emission source (X-rays, visible light, electrons, etc.) and an image creation scheme [6]. Image processing is the technique of exploring and detection the varied pictures out there and providing the desired output within the type of pictures or different elaborate report [7]

\subsection{Some Image Processing Algorithms}

Algorithms for Image Processing Algorithms are the basis for computer Machine Vision and Image Analysis [8]. Employing a theoretical foundation - Image pure mathematics, powerful development tools such as Visual Fortran, Visual C++, Visual Java , Visual Basic and- high-level and efficient base computer, Vision Techniques have been developed by numerous research works. The analysis of the Elementary Image Processing and Machine Vision Algorithms ought to be divided in 5 major teams [9].

- Thinning and Skeletonization Algorithms

- Edge-Detection Techniques

- Digital Morphology

- Grey-Level Segmentation or Thresholding Methods

- Texture 


\subsection{Practises employed for analysing the plants}

There are basically two solutions:

- Real time observation: In this method the field and crops are checked continually and an alarm is signalled as soon as an interest or a disease is sported in few of the plants [10].

- Half-done Classification: When a sickness has to be known amidst many pathologies, it may be the simplest choice to perform the partial classification, in which the regions to be detected square measure being classified as per the explored symptoms and diseases, instead of applying a whole classification into any of the possible diseases. This type of technique is explained in 'Neural Networks' [7].

\subsubsection{Thresholding}

This method tries to discriminate between the affected maize plants, which area unit already affected by the armyworms from the healthy crops victimisation digital pictures. This model categorises the algorithm into 2 methods: image process and image analysis. In the image processing stage, the image is transformed to a gray scale, threshold and filtered to remove artefacts. In the image analysis stage, the whole image is split into 12 blocks. Blocks whose leaf space is less than five-hitter of the whole area unita are discarded. For each remaining blocks, the number of connected objects, representing the diseased and affected regions, is counted [7].

\subsubsection{Neural Networks}

This method tries to discriminate a given sickness from different pathologies that have an effect on the rubber tree leaves. The algorithm will not need any reasonably segmentation techniques. Rather, principal component analysis is applied directly to the RGB values of the pixels of the low resolution (15X15 pixels) pictures of the leaves. Then, the first 2 principal elements square measure fed to the multilayer perceptron (MLP) neural network with one hidden layer, whose output reveals if the sample is infected by some disease of the interest that is familiar, or not [7].

\subsection{Related Works}

A lot of researchers have proposed and comes are with some conventional and non-conventional approaches in helping farmers to detect diseases as when their crops are affected. This paper takes a look at some of these work.

As early as in the 90's an attempt was made by Hetzroni et al. (1994) cited by [1] using neural networks to monitor the health of plants. In their system, they tried to detect zinc, iron and nitrogen deficits by observing lettuce leaves. An analogue video camera was used in image capturing and then digitalized afterwards. The digital image is segmented into background and leaf in the first phase of their algorithm. The required feature (colour and size) are extracted from both the HIS and RGB pictures of the image. These extracted parameters are fed finally into the analysis phase made of neural networks and statistical classifiers, which then determines the condition of the plant [1].

Sena et al. (2003) proposed a method of detecting diseases on leafs using a pre-set threshold value (h), which aims at differentiate among maize plants affected by fall armyworm from healthy employing digital images. Their proposed algorithm was divided into two sections namely the image processing and image analysing. At the processing stage the captured image is transformed to grey scale, filtered and tresholded to removed noise. The image is then divided into twelve block at the analysis stage of their algorithm and blocks with leafs less than $5 \%$ with respect to the total area are thrown away. The number of connected objects (n) signifying the diseased areas is totalled for each remaining block. The plant is concluded to be disease infected if this number is above a set value (threshold), (thus if $n>h$ ) which, after experimental assessment, was set to 10 [11].

Al Bashish et al. (2010) proposed a method which attempts to detect 5 diverse plant diseases. The authors of this paper didn't lay down the types of plants used in their tests, and the images existed in situ. After a pre-processing stage to cleanup the image, a K-means crowding algorithm was applied to divide the image into 4 clusters. From their paper, at least one of the clusters must match to one of the diseases. Afterwards, a number of texture and colour features are extracted from each by means of the supposed Colour Co-Occurrence Technique, which runs with images in the HSI presentation. The features are then fed to a MLP Neural Network with ten (10) concealed layers, which implements the final identification and classification [12].

A mobile enhanced image processing approach for detecting plant leaf diseases was proposed. The research aimed at developing an image recognition system that can recognize crop diseases. The first stage of their methodology was to digitalize the uploaded leaf image by the system user via mobile phone to a remote server. A mathematics morphology is employed to segment these images, then shape, texture and colour features of colour image of disease spot on leaf is extracted, and finally a classification technique of associates functions was used to discriminate between the three types of diseases [13]. The research concluded that there are still numerous techniques that can be employed to better the detection and identification of diseases on plant leafs.

Detecting the Plant Diseases and Issues by Image Processing Technique and Broadcasting was proposed by [7]. The proposal begins with, the image, then an analysis is carried out and eventually, the image is well understood and evaluated. This renders the required target of perceptive plants and their diseases. This forms the key factors of their paper. Their idea was to look at and determine the diseases that attacked the plants, with the help of sensors that use image process techniques to broadcast the captured image to the cloud. In turn, the image can then be viewed in any part of the world [7]. The research concluded that, their approach proves to be an improvement compared to Boesse et al, in 2008 and Pagola et al in 2009.

Skaloudova et al. in 2006 cited by [14] proposed another methodology plan that ascertains the injury caused in leaves by the spider bites and mites. Two stage thresholding was the procedure used for this system. The 1 st $1 / 2$ the leaf in background was targeted within the first a part of the technique. The second part centres on the sorting of the healthy elements of the plants. The final estimation is provided the ratio of the quantity of pixels in injury areas and therefore the number of pixels of the healthy region. This approach was then matched with the outcomes of two unconventional ways and it was realised that the leaf damage index delivered extra advanced results than the opposite ways in contrast supported pigment and light [14].

Dhaygude \& Kumbhar, (2013) proposed a texture statistics technic for plant leaf dieses detection, the developed processing theme consists of four main steps, first a colour 
transformation structure for the input RGB image is created, this RGB is converted to HSI as a result of RGB is for colour generation and his for colour descriptor. Then green pixels area unit cloaked and removed mistreatment specific threshold worth, then the image is segmented and the helpful segments area unit extracted, finally the texture statistics is computed. From SGDM matrices. Finally the presence of diseases on the plant leaf is evaluated [15]. Their research concluded that there is a need to improve or to increase the recognition rate of classification process.

\section{METHODOLOGY}

In disease acknowledgment from an image, the strategic is to extract the characteristic feature of the unhealthy region. According to the disease the choices may vary. The features extracted from the image are, colour, texture etc.

\subsection{The Proposed Approach}

The block diagram shown in Figure 1 depicts the basic technique of the recommended vision-based detection algorithm in this research. Firstly, the images of various plants leaves are going to obtain using a digital camera.

The next stage of the proposed algorithm is to apply the image-processing techniques on the acquired images to extract useful features for onwards analysis.

\section{IMAGE AQUISITION}

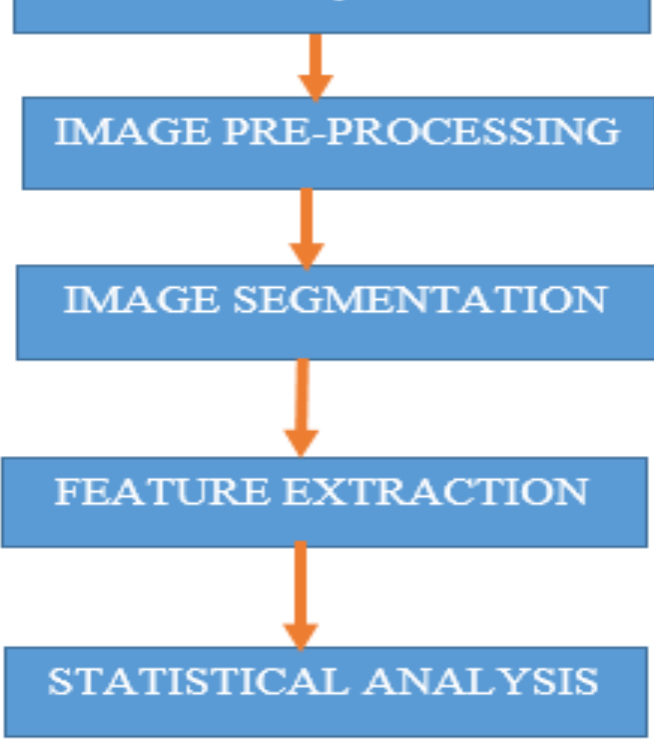

Figure 1. The basic process of the proposed methodology

\subsubsection{The step-by-step process of the proposed system}

The proposed methods are feather divided into subdivision;

- Acquisition of RGB Image

- Convert of RGB image to HSV image format

- Green Pixels Masking

- Masked green pixels removal

- Segmentation of components

- Extract the valuable segment

- Evaluation of texture statistics

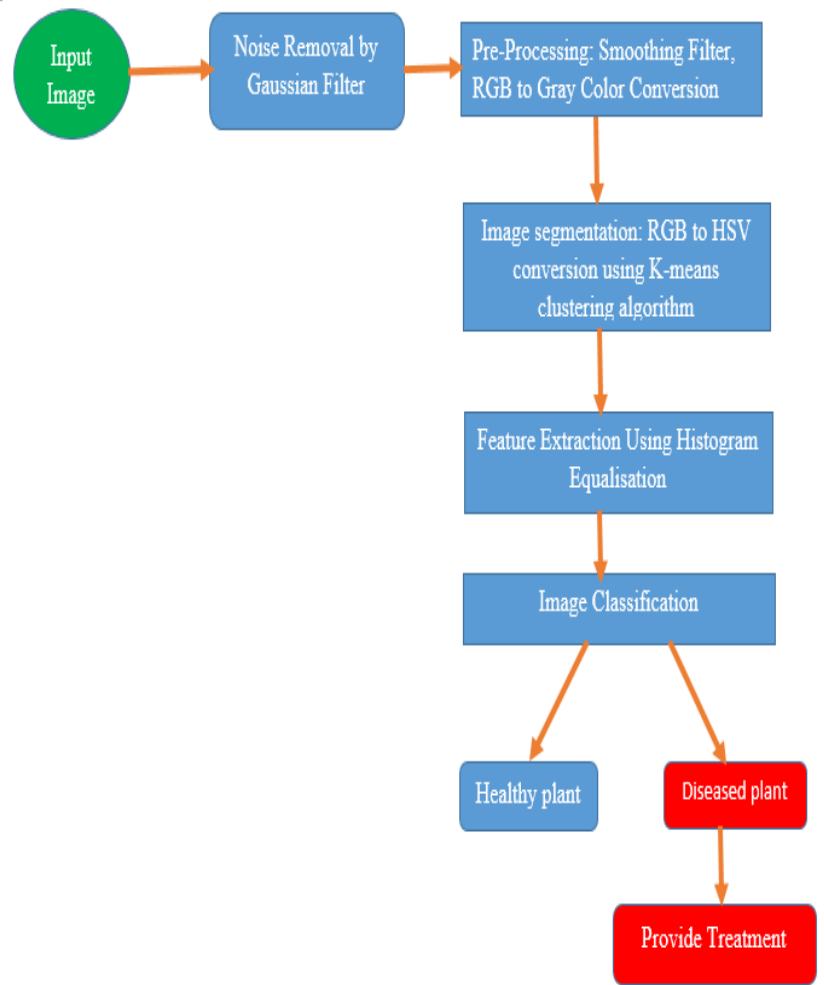

Figure 2 Block Diagram of Plant Leaf Disease Detection

\subsubsection{Image Acquisition}

The images of the plant leaf are captured through the camera in a controlled background and are stored in the JPEG format. Infected leaf is located horizontal on a black background. The leaf is zoomed on so as to make sure that the picture taken contains only the leaf and black background. This image is in RGB (Red, Green and Blue) form. Colour transformation structure for the RGB leaf image is created, and then, a device-independent colour space transformation for the colour transformation structure is applied.

\subsubsection{Image Pre-Processing}

To remove noise in image or other object removal, different pre-processing techniques are considered. Image clipping thus cropping of the leaf image to get the interested image region. Image smoothing is done using the smoothing filter. Image enhancement is carried out for increasing the contrast. The RGB images are converted into the grey images using colour conversion formula as shown in equation (1).

$$
f(x)=0.299 \times R+0.587 \times G+0.114 \times B-----(1)
$$

Then the histogram equalisation which distributes the intensities of the images is applied on the image to enhance the plant diseased leaf images. The cumulative distribution function is used to distribute intensity values.

\subsubsection{Gaussian Filter}

The Gaussian smoothing operator is a 2-D convolution operator that is used to smoothened images and remove detail (higher frequencies) and noise. However, it is similar to the mean filter, but it uses a different kernel that represents the shape of a Gaussian ('bell-shaped') hump.

The general representation of Gaussian filter is shown below:

$$
G(x, y)=1 / 2 \pi \sigma^{2}\left(e^{-\left(\frac{x^{2}+y^{2}}{2 e^{2}}\right)}\right)-
$$




\subsubsection{Image segmentation}

Segmentation means partitioning of image into various part of same features or having some similarity. The segmentation can be done using various methods like otsu' method, kmeans clustering, Boundary and spot detection algorithm, converting RGB image into HIS or HSV model. The RGB image is converted into the HSV model for segmenting using boundary detection and spot detection algorithm. Boundary detection and spot detection helps to find the infected part of the leaf. For boundary detection the connectivity of pixels is considered and boundary detection algorithm is applied.

\subsubsection{Feature Extraction}

Feature extraction plays an important role for identification of an object. In many application of image processing, feature extraction is used. Colour, texture, morphology, edges are the features which can be used in plant disease detection.

\section{RESULTS AND DISCUSSION}

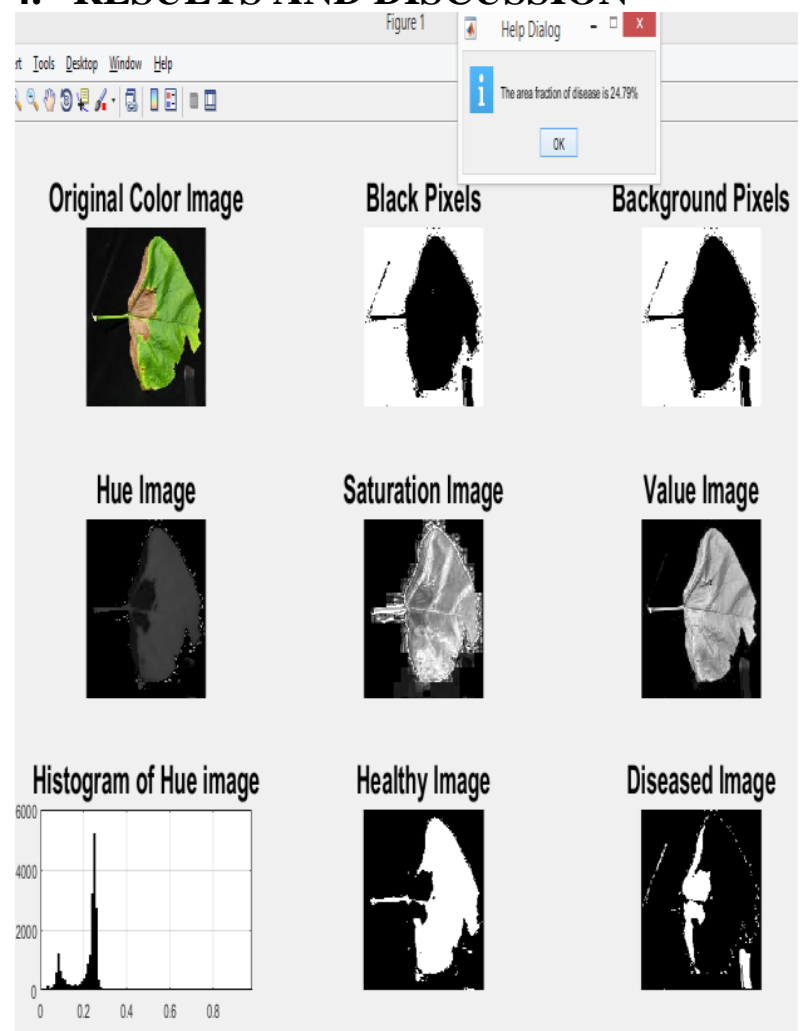

Figure 3 Simulation Results of Plant Leaf Disease System without Filter

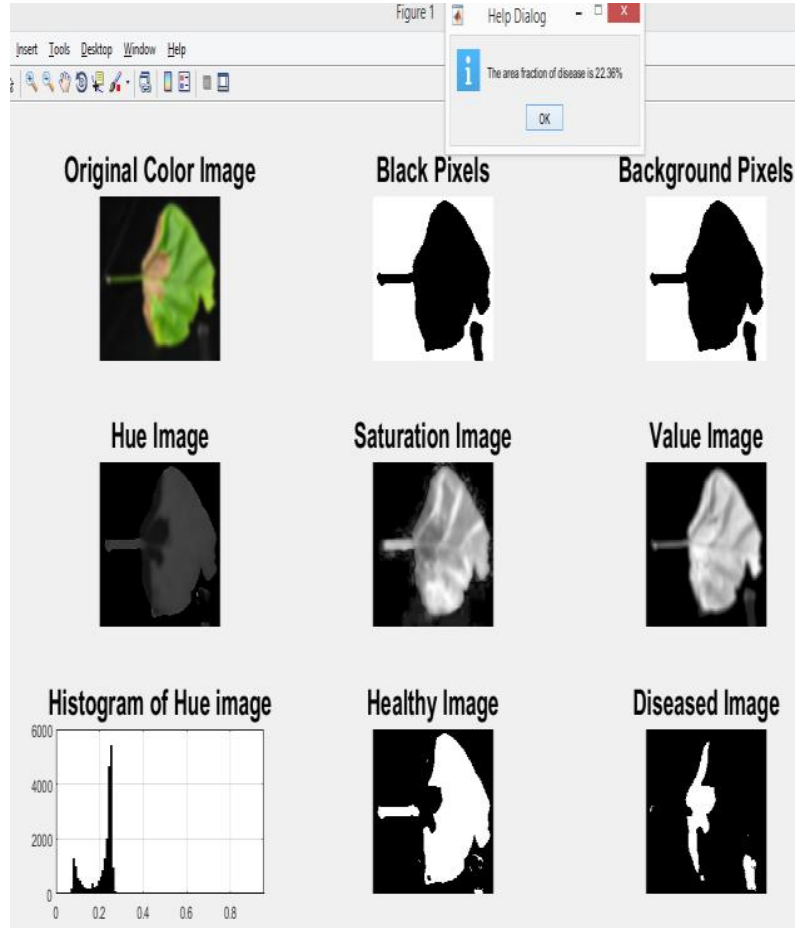

Figure 4 Simulation Results of Plant Leaf Disease Detection System with Filter

Figure 3 shows the simulation result of the propose image processing algorithm for detect plant leaf without the Gaussian filter. Whiles figure 4 shows the output with the Gaussian filter in place.
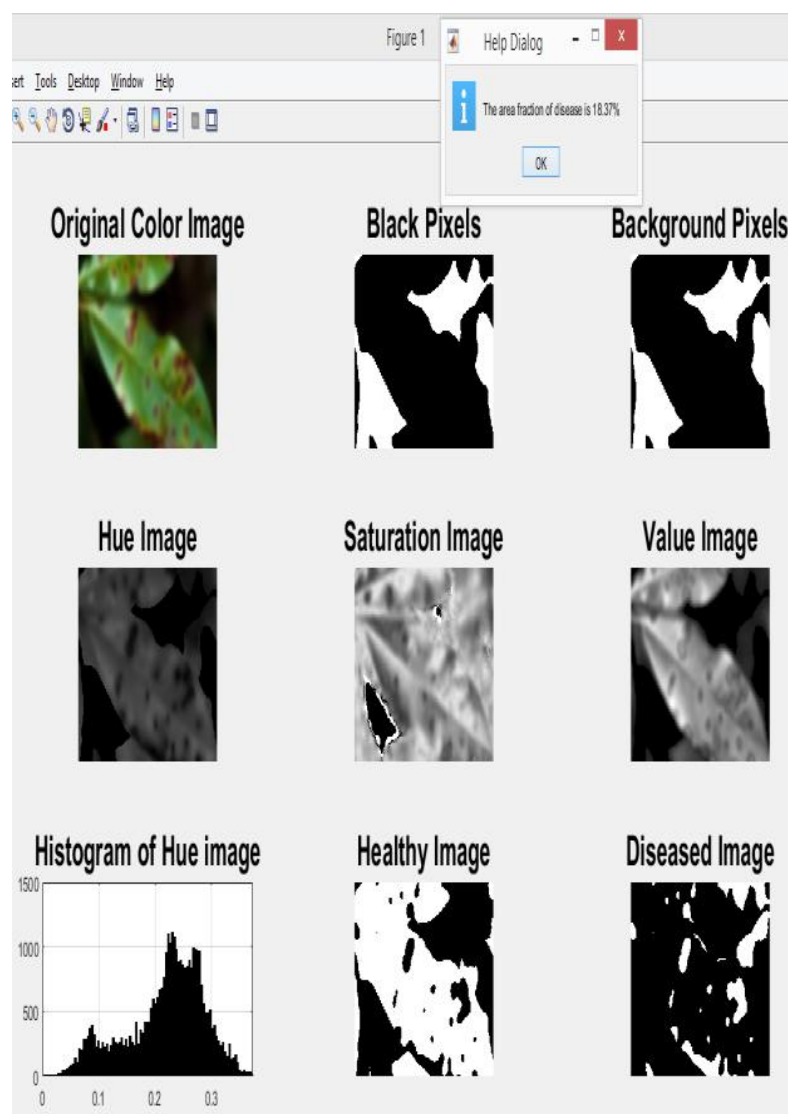

Figure 5 Simulation Results of a Different Plant Species 


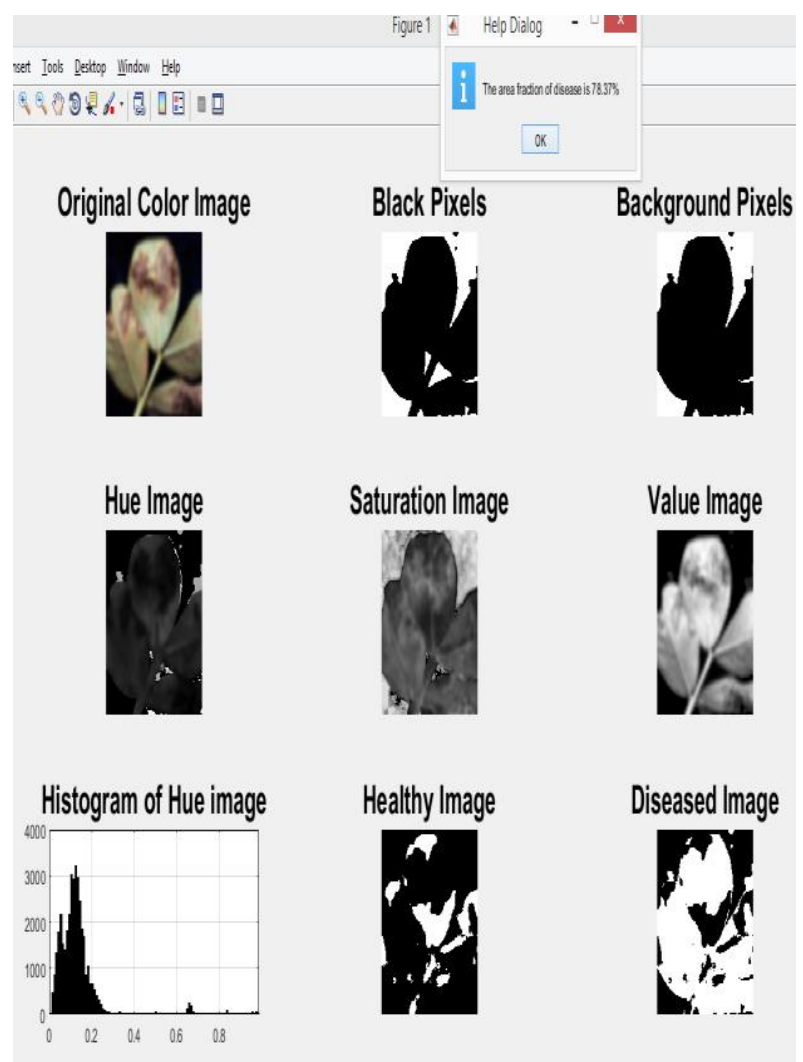

Figure 6 Simulation Results of Multiple Leaves on a Stem

\subsection{Discussion of Results}

The system was tested with image of 259 x 194 pixels. The total samples were 10 from different plant species. The system was simulated in MATLAB. The Graphical User Interface (GUI) result of the leaves are shown in Fig 3, 4, 5 and 6. Figure 3 shows a test results of the first sample without a filter at the pre-processing stage. The system detected the leaf, processed, segmented and displayed the Area Fraction of Disease (AFD) to be $24.79 \%$. The white colours in the healthy and diseased images represent the portions of the leaf that are healthy and diseased respectively. The histogram of the hue image added additional colour to the hue image for proper image visualisation. Gaussian filter was included in the system, and simulation was ran for the same image. It is observed in figure 4 that, smoothened hue, saturation and value images are obtained and the AFD also reduced to $22.36 \%$. This clearly shows that the original image contains some levels of unwanted signals in the form of higher frequencies and noise. Figure 5 and 6 contains different plant leaves. The area fraction of disease are $18.3 \%$ and $78.37 \%$ respectively. The area fraction of disease determines the amount of pesticides to be applied. The higher the AFD the higher the pesticides to be applied

\subsection{Summary of Findings}

MATLAB code for facial recognition was modified and implemented for the plant disease detection system. The system performed better when the Gaussian filter was introduced. This clearly depicts that an image contains some degree of noise which needs to be filtered for successful image processing technique design. The image background has impact on the system performance. When any other background apart from black was used, there was significant effect on the AFD. Hence black background was appropriate for accurate results.

\section{CONCLUSION}

The accurately detection and classification of the plant disease is very important for the successful cultivation of crop and this can be done using image processing. This paper discussed the image processing technique for diseased plant leaf detection. Extraction of features of an infected leaf and the classification of plant diseases can accurately identify and classify various plant diseases and provide the farmer an idea about extend of damage for suitable treatment using image processing techniques. The overall achieved accuracy of the proposed system is higher than $90.96 \%$ in line with the experimental results compared with [2].

\subsection{Feature Work}

The system can work extensively by predicting the type of plant, the kind of disease and recommend the specific pesticides or fungicides to be used, hence recommended for future work.

\section{ACKNOWLEDGMENTS}

Our thanks praise to almighty God and all staff members of STU Electrical/Electronic Department for their various contribution towards this paper.

\section{REFERENCES}

[1] J. G. A. Barbedo, "Digital image processing techniques for detecting, quantifying and classifying plant diseases," Springer Open Journal, pp. 10-12, 2013.

[2] N. Petrellis, "Plant Disease Diagnosis Based on Image Processing, Appropriate for Mobile Phone Implementation," International Conference on Information \& Communication Technologies in Agriculture, Food and Environment, pp. 238-246, 2015.

[3] D. Tilman, K. Cassman, P. Matson, R. Naylor and S. Polasky, "Agricultural sustainability and intensive production practices," Nature, vol. 418, no. 6898, pp. 671-677, 2002.

[4] E. E. Hawkson and T. Ngnenb, "Graphic.com.gh," 2015. [Online]. Available: http://www.graphic.com.gh/news/general-news/ghanaloses-30-per-cent-of-crop-yields-to-pests-diseases.html. [Accessed 2 March 2016].

[5] S. R. Kamlapurkar, "Detection of Plant Leaf Disease Using Image Processing Approach,” International Journal of Scientific and Research Publications, vol. 6, no. 2, pp. 73-76, 2016.

[6] NCSA, "illinois.edu: Image Processing Techniques," 2000. [Online]. Available: http://www.ncsa.illinois.edu/People/kindr/phd/PART1.P DF. [Accessed 10 August 2015].

[7] K. R. Gokulakrishnan and Kapilya, "Detecting the Plant Diseases and Issues by Image Processing Technique and Broadcasting," International Journal of Science and Research, vol. 3, no. 5, pp. 1016-1018, 2014.

[8] A. Martin and S. Tosunoglu, "Image Processing Techniqoes for Machine Vision," Florida International University Department of Mechanical Engineering 10555 West Flagler Street, Miami, Florida 33174, 2000.

[9] G. X. Ritter and J. N. Wilson, Handbook of Computer Vision Algorithms in Image Algebra, CRC Press, 1996. 
[10] G. Anthonys and N. Wickramarachchi, "An image recognition system for crop disease identification of paddy fields in Sri Lanka," International Conference on Industrial and Information Systems (ICIIS) Sri Lanka: IEEE, pp. 403-407, 2009.

[11] D. J. Sena, F. Pinto, D. Queiroz and P. Viana, "Fall armyworm damaged maize plant identification using digital images.," Biosyst Eng, vol. 85, no. 4, p. 449-454, 2003.

[12] D. Al Bashish, M. Braik and S. Bani-Ahmad, "A framework for detection and classification of plant leaf and stem diseases.," 2010 international conference on signal and image processing. IEEE, Chennai, p. 113-118, 2010 .
[13] Y. Sanjana and S. S. J. Ashwath, "Plant Disease Detection Using Image Processing Techniques," International Journal of Innovative Research in Science, Engineering and Technology, vol. 4, no. 6, pp. 295-301, 2015.

[14] M. Zhang and Q. Meng, "Citrus canker detection based on leaf images analysis," In Information Science and Engineering (ICISE), 2010 2nd International Conference. IEEE, pp. 3584-3587, 2010.

[15] S. B. Dhaygude and N. P. Kumbhar, "Agricultural plant Leaf Disease Detection Using Image Processing," International Journal of Advanced Research in Electrical, Electronics and Instrumentation Engineering, vol. 2, no. 1, pp. 599-602, 2013. 